\title{
Editorial
}

\section{Update on cardiopulmonary resuscitation}

Every minute without cardiopulmonary resuscitation (CPR) and defibrillation of a cardiac arrest patient decreases chance of survival between $7-10 \%$. Immidiate action is necessary to save life. Short term survival (post CPR survival for at least 24 hours) is around $38.5 \%$ and successful resuscitation (in hospital CPR for cardiac arrest and discharge from hospital) is seen in $15 \%$ cases as an average and higher rate is seen in younger patient who is less than 70 years.

External cardiac massage and use of defibrillator make change of success of CPR. In 1740 Paris Academy of Science officially recommended mouth to mouth breathing resuscitation for drowning patient. German surgeon Dr. Friedrich Maass described chest compression and effectiveness of effort is seen in artificially produce carotid pulse and constriction of pupil in 1892. Regular research ,patient case studies and opinions of experts is ongoing on to develop it further.

American heart association (AHA) plays important role in this field. They released periodic updates to their CPR and emergency cardiovascular care (ECC) guidelines on five year cycle since 1974. The most recent update publishes in 2015. New recommendations are chest compression should be within 100-120 per minute; compression depth should be at least $5 \mathrm{~cm}$ and allow full recoil of chest after each compression without leaning on chest, minimum pause between compressions. Pause should not exceed more than 10 seconds. Two Ventilation mouth to mouth or by bag valve mask over one second each with adequate chest rise should be done after each 30 compressions. Excessive ventilation should be avoided. Integrated teams of highly trained rescuers need to perform resuscitation as they may use a choreographed approach that accomplishes multiple steps and assessments simultaneously.

During the first few minutes of witnessed cardiac arrest a lone rescuer should not interrupt chest compressions for ventilation. Advanced airway placement in cardiac arrest should not delay initial CPR and defibrillation for VF cardiac arrest. Manual left uterus displacement should be done in cardiac arrest with pregnancy. Naloxone $2 \mathrm{mg}$ intranasal or $0.4 \mathrm{mg}$ intramuscular can be use a patient with cardiac arrest/ abnormal breathing with suspected opioid overdose. Vasopressin is no more needed in CPR and epinephrine should be use as soon as possible in non shockable like pulseless electrical activity case. Shockable rhythm should be aborted by the use of defibrillator (biphasic 120-200 J / monophasic 360J) as soon as possible then after 2 minutes of CPR.

Maximum $\mathrm{FiO}_{2}$ should be used during CPR but it should be adjusted after return of spontaneous circulation. Waveform capnography is used to see effectiveness of CPR. If Low endtidal carbon dioxide $\left(\mathrm{ETCO}_{2}\right)$ is below $10 \mathrm{~mm} \mathrm{Hg}$ attempt should be taken to improve CPR quality. Arterial oxygen saturation should be maintained above $30 \%$. The team member doing compressions should be directed to increase the depth and rate of compressions. Return of the $\mathrm{PETCO}_{2}$ to $35-40 \mathrm{~mm}$ $\mathrm{Hg}$ indicates return of spontaneous circulation. Low end-tidal carbon dioxide $\left(\mathrm{ETCO}_{2}\right)<10 \mathrm{~mm} \mathrm{Hg}$ by quantitative waveform capnography after 20 minutes of CPR in intubated victims is making the decision to terminate resuscitation efforts.

A PETCO $2<10 \mathrm{~mm} \mathrm{Hg}$ indicates that the prognosis for return of spontaneous circulation is poor. Return of spontaneous circulation should be monitored, if systolic blood pressure is less than $90 \mathrm{~mm}$ of $\mathrm{Hg}$ I.V Fluid (normal saline or Ringer's lactate saline) 1-2 litre should be given as bolus and vesopressor should be strated. Targeted temperature management (TTM) should be performed in all comatose adult victims who have spontaneous circulation after cardiac arrest. The target temperature is between $32^{\circ} \mathrm{C}$ and $36^{\circ} \mathrm{C}$ for at least 24 hours. Veno-arterial extracorporeal membrane oxygenation (ECMO) is possible alternative of conventional CPR in patient with refractory arrest with reversible cause. Cardiopulmonary resuscitation is an well organized process. It needs a trained team to be vigilant all the time in hospital who will help patient on time when notified. Every member of hospital also need to be trained on basic cardiopulmonary resuscitation which will is increase quality of care of hospital Patient.

\author{
Dr. Amal Krishna Paul \\ Department of Internal Medicine \\ Khwaja Yunus Ali Medical College \\ Sirajgonj, Bangladesh.
}

